\title{
EXPERIENCIA DE INNOVACIÓN EN EL AULA DESDE LA AUTORREGULACIÓN Y LOS ESTILOS DE APRENDIZAJE
}

\author{
Innovative experience in the classroom from self-regulation and learning \\ styles
}

\author{
María Concepción García Diego \\ e-mail: cgarcia@escuni.es \\ Esther Castañeda López \\ e-mail: ecastaneda@escuni.es \\ José Manuel Mansilla Morales (iD \\ e-mail: jmmansilla@escuni.es \\ Centro Universitario de Magisterio ESCUNI. España
}

\section{RESUMEN}

El presente documento tiene como propósito aportar las conclusiones obtenidas, por el profesorado del Centro Universitario Escuni, sobre las relaciones entre la autorregulación y los estilos de aprendizaje en estudiantes del Grado de Educación Infantil y Educación Primaria. Dichas conclusiones han sido extraídas después de años de investigación sobre la teoría de los estilos de aprendizaje y con la implementación de un modelo metodológico centrado en la autorregulación del aprendizaje. El referente teórico a partir del cual se basa este estudio, se orienta, por un lado en el planteamiento de Kolb (1984) sobre los estilos de aprendizaje, y por otro lado, el modelo de Pintrich (2000) sobre el aprendizaje autorregulado. Las conclusiones obtenidas inciden en la orientación por parte del profesor de los procesos autorregulatorios del aprendizaje desde el conocimiento de los estilos de aprendizaje de los alumnos e, igualmente, desde un ejercicio de autoconocimiento y autoconciencia del alumno, para adquirir mayores competencias académicas y formativas.

PALABRAS CLAVE: estilos de aprendizaje; aprendizaje autorregulado; orientación; innovación metodológica; docentes.

\section{ABSTRACT}

The purpose of this document is to contribute the conclusions obtained by the teachers of Centro Universitario Escuni, on the relations between self - regulation and learning styles in students of the Kinder and Primary Education. These conclusions have been drawn after years of research on the theory of learning styles and with the implementation of a methodological model focused on self-regulation learning. The theoretical reference of this study is based in Kolb's (1984) approach to learning styles and, on the other hand, Pintrich's (2000) model of selfregulation learning. The findings obtained indicate the teacher's orientation of the self-regulating processes of learning from the knowledge of the learning styles of the students and, also, from an exercise of self-knowledge and self-awareness of the student, to acquire greater academic and formative competences.

KEY WORDS: learning styles; self-regulated learning; orientation; methodological innovation; teachers.

Cómo referenciar este artículo / How to reference this article:

García Diego, C. M., Castañeda López, E., \& Mansilla Morales, J. M. (2018). Experiencia de innovación en el aula desde la autorregulación y los estilos de aprendizaje. Tendencias Pedagógicas, 31, 137-148. doi: http://dx.doi.org/10.15366/tp2018.31.008 


\section{INTRODUCCIÓN}

Desde hace algunos años, varios estudios han dejado patente que los alumnos capaces de obtener mejores resultados académicos, también eran estudiantes que seguían un aprendizaje autorregulado (Rosário, Núñez y González-Pineda, 2006; Zimmerman, 2000, 2002; Rosário et al., 2013; Torrano y González-Torres, 2004; De la Fuente, Pichardo, Justicia y García 2008; Zusho, Pintrich y Coppola, 2003; Núñez, González- Pineda, Solano, y Rosário, 2006).

Sin embargo, debemos añadir a este hallazgo, diversas investigaciones que arrojan referencias sobre la insuficiencia de procesos autorregulatorios del aprendizaje en los alumnos universitarios (Boekaerts, Pintrich y Zeidner, 2000). Desde nuestro punto de vista, este déficit podría ser debido a la escasez de formación por parte del profesor sobre aspectos claves del proceso de aprendizaje en los que se invite al alumno a tomar sus decisiones o por la falta de estrategias y conocimiento por parte del alumno de su proceso de aprendizaje, entre otros.

Al visualizar este escenario educativo, tomamos conciencia de una incoherencia didáctica. Tenemos constancia empírica sobre la mejora que provoca la autorregulación para un aprendizaje profundo pero, según nos muestra la literatura, no hay un planteamiento extendido y sistematizado que refuerce y ayude tanto al profesor como al estudiante a mejorar sus procesos de enseñanza y aprendizaje.

Para afrontar esta situación, es imprescindible que la formación de docentes innovadores pase por la propia experiencia vivencial en el proceso de aprendizaje, donde se manejen distintas estrategias didácticas y modelos metodológicos alternativos que incorporen la autorregulación desde el conocimiento del alumno. En este proceso es donde entendemos que el alumnado y futuro educador tiene que adquirir las herramientas necesarias para poder aplicar la metacognición a sus diferentes estrategias de aprendizaje y, así, adquirir las competencias docentes basadas en la reflexión sobre la práctica vivida.

Para favorecer este maridaje contamos con el impulso de los profesores Gallego y Alonso, que han sabido desempeñar un rol fundamental y de gran transcendencia en la comunidad educativa investigando y fomentando intervenciones educativas desde la teoría de estilos de aprendizaje. Una ayuda considerable, especialmente, por toda la literatura y evidencias científicas, como por ejemplo: artículos publicados, congresos internacionales e iberoamericanos y revista monográfica: Journal of learning styles, que han emergido a partir de sus estudios en el panorama educativo de España y de otros países, especialmente de Latinoamérica.

En suma, consideramos de vital importancia en los procesos académicos de enseñanza y aprendizaje reservar un espacio adecuado para fomentar el autoconocimiento por parte del estudiante y para que el profesor acompañe a sus alumnos en su quehacer académico.

Todas estas aportaciones nos llevan a entender que la metodología centrada prácticamente en exclusiva en la enseñanza expositiva como principal recurso en el proceso de enseñanza aprendizaje, tiene que dejar paso al trabajo activo, autónomo y cooperativo del alumno, contando con la tutorización y mediación del profesor y donde sean elementos claves el aprendizaje autorregulado y los estilos de aprendizaje.

Por tanto, a través del análisis de diversos estudios realizados en el Centro Universitario Escuni, se ha considerado la necesidad de extraer los aspectos vinculados a la autorregulación del aprendizaje y reflexionar sobre su relación con los 
estilos de aprendizaje, la personalidad y el rendimiento académico en nuestros alumnos. Una vez realizada esta reflexión, se plantean una serie de orientaciones a través de las cuales se espera aportar recursos facilitadores para la adecuada implementación de esta metodología en las aulas.

\section{EL APRENDIZAJE AUTORREGULADO}

La línea de investigación sobre los procesos autorregulatorios cobra fuerza en España en la década de los 90 con los estudios de Beltrán (1993), Mayor, Suengas, González (1993) y Pozo (1990) y Monereo (1994) entre otros. Ahora bien, no debemos olvidar que ya en la década de los 70 , se estudiaba la implicación de la regulación en los procesos de aprendizaje de los estudiantes.

Nos encontramos ante un constructo relevante ya que ha recibido un gran tratamiento en la literatura específica sobre el aprendizaje pero que conlleva grandes dificultades en su conceptualización (Pintrich, 2000). La autorregulación es entendida como un constructo con límites porosos a otros tradicionales que se consideran exponentes del aprendizaje. Según García Martín (2012), se concibe "como la acción reguladora que una persona ejerce en los distintos momentos de su proceso de aprendizaje".

La autorregulación consiste en un proceso activo en el que los alumnos se hacen protagonistas de su propio aprendizaje, lo interiorizan y se adueñan de los objetivos que se persiguen en él (Pintrich, 2000). Se erige, así, en un proceso consciente que ayuda al estudiante a ser proactivo en su aprendizaje y por tanto entendemos que partir del estilo de aprender, facilitará el control y la regulación de las estrategias que permiten al alumno alcanzar las metas propuestas.

Al revisar la literatura, nos encontramos diferentes teorías que pretenden explicar de qué manera se produce la autorregulación (Zimmerman y Schunk, 2001): operante, fenomenológica, del procesamiento de la información, volitiva, vigotskiana, cognitivo-constructivista. En nuestra práctica educativa nos hemos centrado en el modelo de componentes motivacionales y cognitivos de Pintrich (2000), inspirado en Bandura. Se trata de un marco similar al de Zimmerman, elaborado en 1998 y revisado en el 2000 en su concepción, ya que explica el aprendizaje autorregulado como un proceso cíclico caracterizado por una sucesión de fases en la que influye el contexto social.

Cuando Pintrich (2000) define la autorregulación lo entiende como un proceso activo y constructivo. Resalta la importancia de que los estudiantes fijen las metas y monitoricen sus cogniciones, motivaciones y conductas determinadas. Incluso las características contextuales y el ambiente pueden ser controladas y modificadas por el propio aprendiz en función de las propias metas. El proceso de aprendizaje lo fragmenta en cuatro fases: a) planificación, b) autoobservación, c) control d) evaluación. Fases secuenciadas no de forma jerárquica o lineal, sino que se pueden dar de forma dinámica y simultánea.

En suma, la autorregulación constituye un proceso fundamental para mejorar la calidad en el aprendizaje que gana sentido si se contempla vinculada a la teoría de estilos de aprendizaje.

\section{ESTILOS DE APRENDIZAJE}

Tal y como hemos expuesto anteriormente, si entendemos la autorregulación como un proceso en el que intervienen variables cognitivas, contextuales, emocionales 
y volitivas, podemos comprobar que guarda una estrecha vinculación con la conceptualización de estilos de aprendizaje de Keefe (1998, citado por Alonso, 2002). Este autor facilita una definición completa y compleja de los estilos de aprendizaje indicando que son "los rasgos cognitivos, afectivos y fisiológicos, que sirven como indicadores relativamente estables, de cómo los discentes perciben, interaccionan y responden a sus ambientes de aprendizaje".

Por otro lado, debemos considerar que según Kolb (1984, citado por Alonso, Gallego y Honey, 2002), un auténtico aprendizaje requiere de cuatro fases: experimentar, reflexionar, teorizar y poner en práctica. Por lo tanto, conviene dotar las experiencias de aula con actividades y recursos variados para que todos los estudiantes adquieran el aprendizaje teniendo en cuenta su estilo de aprendizaje. Ahora bien, igualmente les debemos orientar para que a partir de su estilo preferente puedan potenciar el resto de las fases que completan el modelo cíclico del aprendizaje. Los ámbitos que incorpora la teoría de estilos de aprendizaje tienen en cuenta que "el discente más capacitado será aquel que sea capaz de aprender en cualquier situación que se le presente. Para esto, necesita un buen nivel de preferencia en todos los estilos de aprendizaje" (Alonso, 2002, p. 157).

Si tenemos en cuenta estos supuestos, la rueda de aprendizaje de Kolb (1984) nos recuerda al modelo de Pintrich (2000). La fase de preparación continúa con la de autoobservación centrada en la reflexión, seguida de un momento de control ajeno a la ambigüedad, de estudio y análisis y finaliza con la autoevaluación que en el caso del ciclo de aprendizaje sería una puesta en práctica y valoración de los resultados, y que a su vez constituiría el punto de partida para volver a planificar e iniciar una nueva acción (González, 2001). En cualquiera de los dos modelos no hay un momento universal de partida, va en función del sujeto y del contexto.

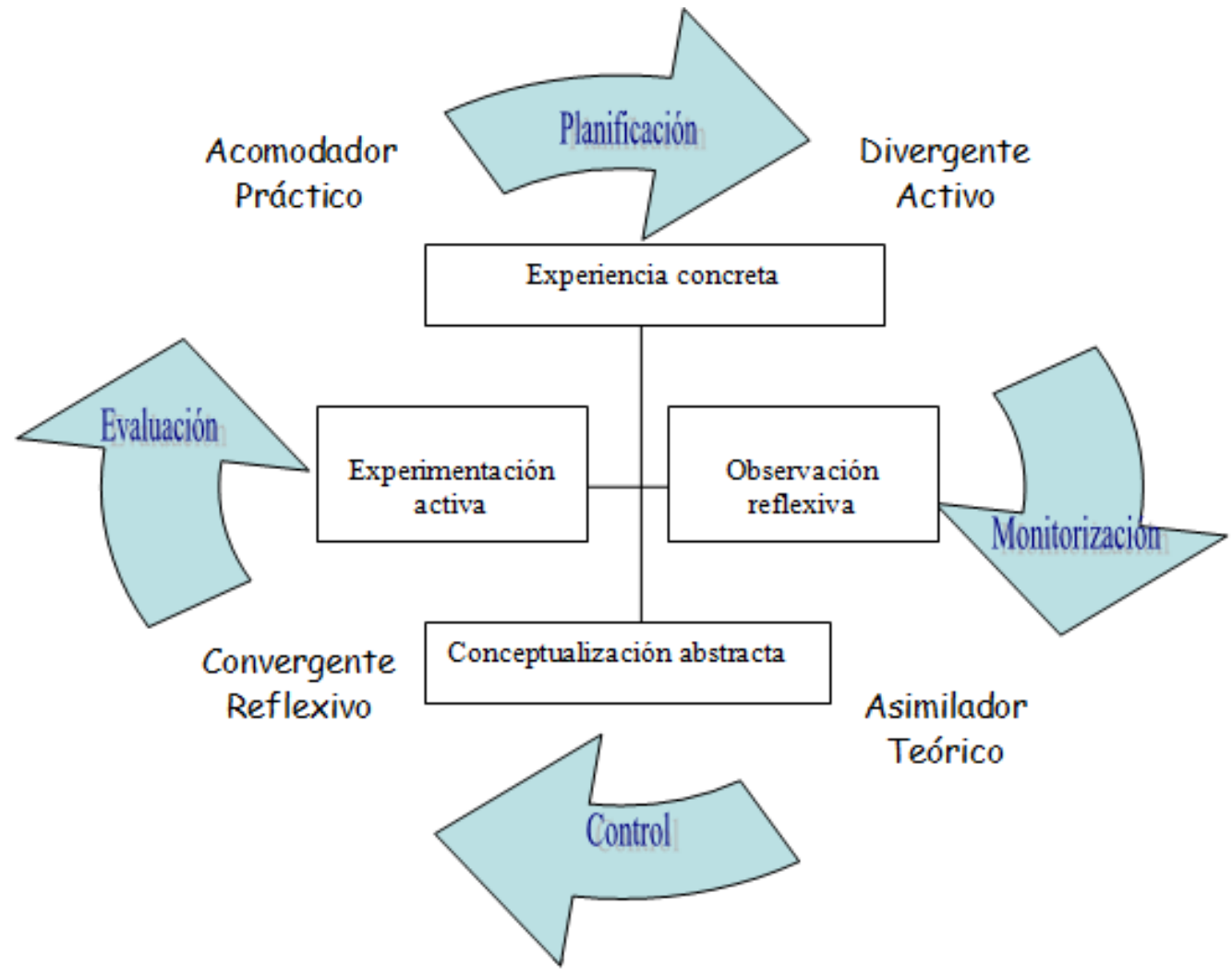


Gráfico 1.- Similitud entre la rueda de aprendizaje de Kolb y las fases de autorregulación de Pintrich.

La reflexión que en este documento presentamos analiza los estilos de aprendizaje siguiendo el modelo de Honey y Mumford (1986) inspirado en el modelo de Kolb (1984), continuado por Alonso (1992), donde se analizan los siguientes estilos: 1) activo, 2) teórico, 3) reflexivo y 4) pragmático. Alonso añade una clasificación de características que acompañan a cada estilo en dos niveles, en el primero sitúa las denominadas características principales que se han obtenido a partir de las puntuaciones significativas según el análisis factorial realizado a partir de 1.371 encuestas; en el otro nivel, agrupa otra serie de características que completan cada uno de los estilos de aprendizaje.

A pesar de que no es posible encontrar personas con estilos puros sino perfiles de estilos de aprendizaje (Alonso, 1992), sí presentan predominio por uno de ellos (activo, reflexivo, teórico y pragmático). Las características que acompañan a cada estilo son las siguientes: 1) en el activo sobresalen las características de animador, improvisador, descubridor, arriesgado o espontáneo; 2) en el estilo reflexivo, el ser ponderado, concienzudo, receptivo, analítico y exhaustivo; 3) en el estilo teórico destaca el ser metódico, lógico, objetivo, crítico y estructurado; 4) en el estilo pragmático, las características de experimentador, práctico, directo, eficaz y realista.

La investigación de García Diego (2011) corroboró las características asociadas a estos estilos de aprendizaje aplicando el Cuestionario de Estilos de Aprendizaje CHAEA, cruzando estos resultados con los obtenidos en el test de personalidad 16PF-5. A través de esta interacción, obtuvo una serie de características que vinculó a cada uno de los estilos. Así, el estilo activo se presenta como "dominante, asertivo, tenso, enérgico, impaciente e intranquilo, independiente, crítico, gusto por la polémica, seguidor de sus impulsos, poco autocontrolado, inconformista, poco cumplidor con las norma, dependiente del grupo y tolerante con el desorden"; el estilo reflexivo como "atento a las normas, cumplidor con los trabajos y los plazos, aprensivo, inseguro y preocupado, perfeccionista, organizado y disciplinado, autocontrolado, serio y reprimido, tímido, temeroso y cohibido, relajado, plácido y paciente, acomodaticio y aceptador de acuerdos"; el estilo teórico: "Atento a las normas, cumplidor y formal, autosuficiente, individualista y solitario, perfeccionista, organizado y disciplinado, autocontrolado, frío, impersonal y distante, serio, reprimido y cuidadoso, introvertido y socialmente inhibido"; y por último, en el estilo pragmático encontró características como "tenso, enérgico, impaciente e intranquilo, extravertido, social y participativo, perturbable, con ansiedad, independiente, crítico y con gusto por la polémica" (García Diego, Mansilla y Molina, 2016 pp.83-84).

Debemos reseñar que en ningún caso podemos interpretar las características de los diferentes estilos como mejores que otras, es decir, como que un estilo es mejor que otro. Se trata de predisposiciones genéticas, contextuales, cognitivas y sociales, y las personas nos encontramos con mayor o menor predisposición hacia un estilo en cada una de las fases del aprendizaje. Por este motivo consideramos importante que el alumno pueda experimentar los cuatro estilos de aprendizaje, ya que de esa manera será más competente en cualquier momento de su proceso de aprendizaje.

\section{CONTEXTUALIZACIÓN DE LA EXPERIENCIA}


EI Centro Universitario ESCUNI está implementando desde el curso 2015-2016 una nueva metodología basada en el aprendizaje autorregulado, en la formación inicial de Grado de Educación Infantil y de Educación Primaria.

Este ha sido el momento de arranque de una nueva propuesta pedagógica, que se puede considerar como el momento de inmersión en una experiencia piloto con los alumnos de 1er curso y por tanto el momento más adecuado para realizar las consideraciones que en este documento se incluyen. El plan de actuación consistió en cuestiones básicas y centrales para nuestro proyecto de investigación: reuniones informativas generales, consulta de materiales, reuniones semanales con el profesorado, etc.

Por ello, como parte de la actividad investigadora del centro, se pretende analizar pormenorizadamente los procesos de autorregulación en el aprendizaje desde el conocimiento del alumno, aprovechando todas las oportunidades que nos brinda la teoría de estilos de aprendizaje, las características de la formación inicial del futuro docente y el potencial del trabajo tutorizado por el profesor y administrado en el aula de forma individual y/o grupal.

Por otro lado, ha sido a partir del curso 2011/2012 cuando se ha comenzado a trabajar con rigor sobre la teoría de los estilos de aprendizaje con nuestros estudiantes. Se comenzaron a realizar investigaciones de tipo no experimental con un diseño ex post facto ya que se ha efectuado sin tener control sobre las variables estudiadas y sin haber sido manipuladas. En todos los casos han sido realizadas una vez producidos los acontecimientos que se pretendían estudiar.

Se realizaron dos estudios trasversales con estudiantes de nuevo ingreso y uno longitudinal. El primero (García Diego, 2011) con una muestra de 210 estudiantes de los alumnos de Grado de Educación Infantil y de Educación Primaria. El segundo de ellos con una muestra de 105 estudiantes (García Diego, Mansilla, Morales y Ceniceros, 2016). El último estudio de corte longitudinal (García Diego, Mansilla y Molina, 2016) contó con una muestra de 141 alumnos (ya que hubo mortalidad experimental y no se obtuvieron datos de 67 alumnos al finalizar el estudio, de los 210 iniciales), realizado con la misma muestra de estudiantes en dos momentos diferentes de su formación inicial: primer y último curso.

En estos estudios se analizaron los estilos de aprendizaje y su relación con la personalidad y el rendimiento académico. En ellos, al observar variables que se presuponen en la autorregulación del aprendizaje, encontramos una vía a través de la cual reflexionar y analizar cómo la autorregulación se relaciona con ellos.

El instrumento de medida que se aplicó para conocer los estilos de aprendizaje fue el Cuestionario Honey- Alonso de Estilos de Aprendizaje, CHAEA, (Alonso, Gallego y Honey, 1997). Este instrumento presenta como aspecto destacable, la posibilidad de que el propio alumno complete su perfil, profundizando así en el diagnóstico del autoconocimiento.

Por otro lado, para valorar la personalidad se empleó la adaptación española del Test de Personalidad 16PF-5 (Cattell y Cattell, 1995). Mide las mismas 16 escalas primarias del 16PF: afabilidad, razonamiento, estabilidad, dominancia, animación, atención a las normas, atrevimiento, sensibilidad, vigilancia, abstracción, privacidad aprensión, apertura al cambio, autosuficiencia, perfeccionismo y tensión. Y las cinco escalas superiores denominadas dimensiones globales: extraversión, ansiedad, dureza, independencia y autocontrol. 
La variable de rendimiento académico se calculó en función de todas las calificaciones que obtuvieron los alumnos en las diferentes asignaturas en el curso académico en el que estuvieran realizando sus estudios.

Una vez recogida la información, se introdujo en una base de datos con el programa SPSS, versión 13.0 para Windows, para su tabulación y análisis. Los estudios que se llevaron a cabo incluyeron la estadística descriptiva para los estilos de aprendizaje y el análisis inferencial para obtener la correlación entre las variables de estudio, concretamente el coeficiente de correlación de Pearson con un nivel de significación de 0,05.

\section{CONCLUSIONES DE LAS INVESTIGACIONES REALIZADAS CON LOS ESTUDIANTES}

Atendiendo a los resultados académicos, observamos que los estudiantes de Grado de Educación tanto de Infantil como de Primaria que obtienen mejores resultados académicos, se relacionan con el estilo de aprendizaje teórico, sin embargo, los estudiantes que obtienen el peor rendimiento académico se identifican con el estilo activo. Estos resultados van en la misma línea que los encontrados por López y Silva (2009) y Ruiz, Trillos y Morales (2006).

Este hallazgo nos llama especialmente la atención, ya que debemos tener en cuenta que la mayor concentración de alumnos corresponde al estilo activo, si es cierto que algunos de ellos sin tener puntuaciones elevadas (García Diego, 2011). Datos que no sorprenden ya que las investigaciones de González Tirados (1985) y de Alonso (2002) recogían la predominancia del estilo activo en las carreras de Humanidades, donde se incluían los estudios de Magisterio.

Por otro lado, al analizar las relaciones entre la personalidad y los estilos de aprendizaje en nuestros alumnos (García Diego, 2011), identificamos que el estilo activo se vincula con los rasgos de atrevimiento, extraversión, apertura al cambio e independencia, sin embargo, presenta poca atención a las normas, al perfeccionismo y al autocontrol. Tanto el estilo reflexivo como el teórico puntuaron alto en atención a las normas, perfeccionismo y autocontrol, no así en la escala de animación. En el estilo pragmático sólo se identificaron correlaciones positivas en tensión, extraversión, ansiedad e independencia.

Por tanto, parece que hay una estrecha relación entre características de la personalidad y características de los estilos de aprendizaje. Esto nos hace pensar que el acompañamiento en los procesos de aprendizaje debe tener en cuenta que el autocontrol, el perfeccionismo y la atención a las normas son elementos que convergen en el rendimiento académico.

Estos planteamientos entran en sintonía con estudios sobre la autorregulación como los de Pintrich (2000), Zimmerman y Schunk (2001). Estos autores exponen que algunas características de personalidad contribuyen, de diferente manera, a facilitar el proceso autorregulatorio en el estudiante. Otros estudios como el que Reyes (2003) da a conocer sobre los trabajos de Aliaga, muestran una correlación entre el rendimiento en las asignaturas y los rasgos de personalidad de animación, respeto por las normas y sensibilidad, medidos a través del 16 PF de Cattell - Forma A. Estos estudios presentan resultados similares a los encontrados por Barrera, Donolo, y Rinaudo (2010) que identificaron que los estudiantes con mayor rendimiento son los que tienen mayores recursos de procesamiento y autorregulación de su aprendizaje. 
En este sentido, en nuestra investigación (García Diego, 2011) también se estudió el predominio de dos o más estilos con las dimensiones de personalidad, donde se halló que el perfeccionismo y el autocontrol correlacionaron significativamente. Se trata, pues, de un alumnado organizado, controlado y disciplinado. Es decir, según estos datos, la organización y el autocontrol están apuntando de nuevo hacia buenos resultados.

Al realizar el estudio longitudinal se esperaba encontrar diferencias significativas en la predominancia del estilo entre el primer y cuarto curso, incluso por las propias materias, como por ejemplo en el caso de la elaboración del Trabajo Fin de Grado o por el período de prácticas que cursan durante un intervalo de tiempo considerable en un centro escolar. Sin embargo, los cambios en los estilos de aprendizaje estudiados parecen depender del estilo predominante de partida. Este hecho se observa para el estilo activo, reflexivo y pragmático, sin embargo, el estilo de aprendizaje teórico es el que con mayor frecuencia cambia al estilo pragmático en el último año de carrera. Incluso, los datos indican que aunque es difícil anticipar el comportamiento futuro de los estilos de aprendizaje (García Diego, Mansilla y Morales, 2016), el estilo que mejor se prevé es el que corresponde al estilo de aprendizaje activo.

Teniendo en cuenta los resultados presentados anteriormente y desde nuestro punto de vista, la implementación de un modelo pedagógico centrado en el aprendizaje autorregulado debe considerar las características de los estilos de aprendizaje con el objetivo de obtener el máximo rendimiento del estudiante. Así, la orientación cobra un matiz más individualizado para guiar al alumno, a la vez que le brinda un mayor número de posibilidades en el camino hacia un productivo proceso de aprendizaje.

\section{ORIENTACIONES PARA FAVORECER LA AUTORREGULACIÓN A PARTIR DEL ESTUDIO DE LOS ESTILOS DE APRENDIZAJE}

A continuación, ofrecemos orientaciones que desde nuestra acción, reflexión, teorización y puesta en práctica, pueden resultar interesantes para la comunidad educativa que esté incorporando el aprendizaje autorregulado y sobre la teoría de los estilos de aprendizaje en el aula.

Somos conscientes de que los aspectos que planteamos no son fáciles de acometer, pero conviene tenerlos presente y considerarlos a la hora de implementar una metodología basada en el aprendizaje autorregulado a partir de los estilos de aprendizaje. Como se podrá observar, esto supone cambios significativos en la forma de actuar y de pensar del profesorado y en la estructura organizativa de cualquier centro educativo que quiera desarrollarlo adecuadamente, y por tanto entendemos que a veces pueden ser desestabilizadores de una tradición didáctica muy enraizada.

Pasamos, pues, a plantear un compendio de planteamientos extraídos del análisis de la literatura y de la práctica docente diaria y, en algún caso, requisitos imprescindibles para la incorporación del nuevo modelo pedagógico en el centro.

1. Es necesario configurar el diseño del recurso temporal y su repercusión en el recurso espacial, fundamentalmente centrado no solo dentro del aula convencional sino haciendo uso de los diferentes espacios del centro educativo. Aumentan, así, las posibilidades de movimiento autónomo del alumno, en función de las tareas que está desempeñando, de las metas a conseguir, de las directrices propias y del profesor, y de los recursos que tiene a su 
disposición. Es fundamental contar con espacios iluminados, ventilados y que posibiliten tanto el aprendizaje individual y grupal.

2. El recurso temporal posibilita un mayor acompañamiento del alumno por parte del profesorado dentro de su materia. Concentrar en sesiones amplias el tiempo dedicado a las asignaturas favorece un escenario de trabajo favorecedor. Esto es un requisito ineludible, ya que las unidades convencionales de 50 ó 60 minutos por asignatura no permiten adentrarnos en procesos de trabajo que exijan profundidad ya que son más lentos los procesos cuando el alumno "hace". Por este motivo, un elemento clave es facilitar tiempos amplios para que el alumno pueda ser constructor de su aprendizaje de forma individual, grupal, con acompañamiento más o menos directo por parte del profesor o de un grupo de profesores.

3. Debemos asumir un cambio de rol en el profesor, de transmisor a mediador, es decir, en nuestro caso a guía del aprendizaje autorregulado del alumno. No se contempla de forma exclusiva y dominante el qué se enseña sino el cómo se aprende. Por lo tanto, la presencia del modelo expositivo no se pierde, pero se minimiza, a favor de la actividad del alumno. Las neurodidácticas nos lo están fundamentando todos los días, no debemos desestimar sus aportaciones. La mera transmisión provoca una superficialidad en el aprendizaje que no permite operativizar lo esencial.

4. Cambia la concepción del control: se da una sensación de pérdida de control por parte del profesorado, sobre todo en los primeros momentos de incorporar el nuevo modelo pedagógico. El silencio es menor y el movimiento mayor. Esto puede ocasionar sensaciones de incomodidad en algunos profesores acostumbrados a contextos más expositivos y de transmisión más controlada. También va en función del estilo de enseñar de cada uno de los docentes, ahora bien, si queremos que un alumno sea activo, reflexivo, teórico y que sea capaz de poner en práctica lo aprendido, los escenarios pedagógicos serán silenciosos o llenos de expresión y movimiento en función de la tarea a realizar.

5. Se desplaza el peso que prácticamente tenía en exclusiva el contenido de las materias hacia la valoración del procedimiento. Las tecnologías de la información y comunicación abrieron ya hace décadas estos nuevos desafíos pero que aún hoy son considerados como deficiencias. Aprender es favorecer nuevas conexiones neuronales que se activan si los conocimientos a aprender están vinculados a algo significativo o a conocimientos previos. El aprendizaje es lento, además requiere un ejercicio de atención selectiva y sostenida al que hay que prestar atención.

6. Se mantiene una tutoría académica en los departamentos para los estudiantes que la reclamen o que sea solicitada su presencia por parte del profesor. Ahora bien, gana fuerza, en este modelo, los momentos de trabajo autorizado y la tutoría en el aula. El profesor en el aula orienta, explica, facilita, retroalimenta y guía de forma grupal e individual pero de forma también más natural. Es requisito que estos acompañamientos se realicen generando ambientes de confianza y donde se atienda a los aspectos emocionales de los estudiantes.

7. Un logro en el proceso de aprendizaje autorregulado es mejorar la comunicación clara de objetivos y la constatación de su interiorización por parte de los alumnos. Igualmente, diseñar una secuencia de tareas y de un plan de tiempos regulado y administrado por el alumno. No son buenas las prisas en estas fases del proceso de enseñanza. Esto nos obliga a prestar atención a todos los estudiantes y de forma especial al estudiante con estilo activo. Pueden requerir una ayuda para gestionar su aprendizaje desde la actividad, desde los desafíos desde el liderazgo pero facilitándole un acompañamiento 
que le facilite la regulación de su propio proceso. Como expone García Martín (2012), "un alumno difícilmente será autorregulado académicamente si no posee conocimiento sobre sí mismo, sus emociones y sus estrategias de aprendizaje" (p.205).

8. Facilitar recursos diversos para la búsqueda de información que tengan en cuenta los diferentes estilos de aprendizaje. Utilizar espacios y tiempos variados y adecuados para desarrollo de tarea individual y/o grupal y en función de ella. Fomentar momentos de reflexión sobre su actuación en el momento de aprender y explicitar las creencias y sus motivaciones. Hacer de la práctica del portafolios, solicitado desde varias asignaturas e incluso como trabajo a entregar después del periodo de prácticas en los colegios de infantil y primaria, un verdadero instrumento de autorregulación.

Un aprendiz docente debe incorporar en su repertorio habilidades y competencias que le faciliten ser activo, reflexivo, teórico y pragmático según el momento y el contexto en el que se encuentre. Si entendemos al maestro como un agente educativo activo, creativo, innovador y reflexivo, la implementación de procesos autorregulatorios en su intervención educativa pasa por brindar herramientas para que el estudiante se conozca mejor, planifique y administre estrategias determinadas en función de la tarea a realizar, realice adecuadamente la toma de decisiones en su aprendizaje y en definitiva, se forme para un aprendizaje a lo largo de toda la vida.

\section{REFERENCIAS BIBLIOGRÁFICAS}

Alonso, C. M. (1992). Análisis y Diagnóstico de los Estilos de Aprendizaje en Estudiantes Universitarios, Tomos I y II, Tesis Doctoral, Madrid: Universidad Complutense.

Alonso, C. M. Gallego, D. y Honey, P. (2002). Los Estilos de Aprendizaje. Procedimientos de diagnóstico y mejora, Bilbao: Ediciones Mensajero.

Alonso, C. M., Gallego, D.J. y Honey, P. (1997). Los estilos de aprendizaje. Procedimientos de diagnóstico y mejora. España: Ediciones Mensajero

Barrera, M., Donolo, D. y Rinaudo, M. (2010). Estilos de aprendizaje en alumnos universitarios: peculiaridades al momento de aprender. Revista de Estilos de Aprendizaje, 6(6), 1-27.

Beltrán, J. (1993). Procesos, estrategias y técnicas de aprendizaje. Madrid: Síntesis.

Boekaerts, M., Pintrich, P. R., \& Zeidner, M. (Eds.). (2000). Handbook of self-regulation. San Diego: Academic Press.

Cattell, R. \& Cattell, H. (1995). Personality Structure and the New Fifth Edition of the 16PF. Educational and Psychological Measurement, 55 (6), 926-937. https://doi.org/10.1177/0013164495055006002

De la Fuente, J., Justicia, F., Berbén, A.B., y Pichardo, M.C. (2008). Enfoques de aprendizaje, autorregulación y rendimiento en tres universidades europeas. Psicothema, 20(4), 705-711.

García Diego, M.C. (2011). Inteligencia, Personalidad y Estilos de Aprendizaje. UNED: Tesis Doctoral.

García Diego, Maㅡ C., Mansilla Morales, J. M. y Ceniceros Estevez, J. C. (2016). Los estilos de aprendizaje y la autonomía en el universitario. Actas VII Congreso Mundial Estilos de Aprendizaje. Braganza. En https://cloud.ipb.pt/f/6330d8d509/?dl=1 
García Diego, Mã. C.; Mansilla Morales, J. M. y Molina Ferragut, E. (2016). La relativa estabilidad de los estilos de aprendizaje en estudiantes de Magisterio: Estudio longitudinal. Journal of Learning Styles, abril, vol. 9, №17. ISSN: 2232-8533. En http://learningstyles.uvu.edu/index.php/jls/article/view/286/206

García Martín, M. (2012). La autorregulación académica como variable explicativa de los procesos de aprendizaje universitario. Revista de curriculum y formación del profesorado, 16 (1). Recuperado el 12 de julio de 2012 de: http://www.ugr.es/ recfpro/rev161ART12.pdf

González Tirados, R. M. (1985). Influencia de la naturaleza de los estudios universitarios en los estilos de aprendizaje de los sujetos. Tesis Doctoral. Madrid: Universidad Complutense.

González, A. (2001). Autorregulación del aprendizaje: una difícil tarea. IberPsicología, 6(1), 30-67.

Honey, P. y Mumford, A. (1986). Using our Learning Styles. Berkshire, United Kingdom: Peter Honey.

Kolb, D. (1984). Experiential learning experiences as the source of learning development. Nueva York: Prentice Hall.

López, M., y Silva, E. (2009). Estilos de aprendizaje. Relación con motivación y estrategias. Revista Estilos de Aprendizaje, 4(4), Recuperado de http://www.uned.es/revistaestilosdeaprendizaje/numero 4/Artigos/lsr 4 articul o 3.pdf

Mayor, J.; Suengas, A. y Gonzalez, J. (1993): Estrategias metacognitivas. Aprender a aprender y aprender a pensar. Madrid. Síntesis.

Monereo, C. (coord.) (1994) Estrategias de enseñanza y aprendizaje: formación del profesorado y aplicación en la escuela. Barcelona: Graó.

Núñez, J. C., González-Pineda, J., Solano, P. y Rosário, P. (2006). Evaluación de los procesos de autorregulación mediante autoinforme. Psicothema, 18(3), 353358.

Pintrich, P. (2000). The role of goal orientation in self-regulated learning. En Boekaerts, M., Pintrich, P. Zeidner, M. (Eds.). Handbook of Self-regulation. San Diego: Academic

Press. https://doi.org/10.1016/B978-012109890-2/50043-3

Pozo, J.I. (1990). Estrategias de aprendizaje. En C. Coll; J. Palacios y A. Marchesi: Desarrollo psicológico y educación, II. Madrid: Alianza.

Reyes, Y. N. (2003). Relación entre el rendimiento académico, la ansiedad ante los exámenes, los rasgos de personalidad, el autoconcepto y la asertividad en estudiantes del primer año de psicología de la UNMSM. Tesis Doctoral, En http://sisbib.unmsm.edu.pe/bibvirtual/tesis/salud/reyes t y/contenido.htm

Rosário, P., Núñez, J. C. y González-Pineda, J. (2006). Comprometerse com o estudar na Universidade: "Cartas do Gervásio ao seu Umbigo». Coimbra: Almedina.

Rosário, P., Núñez, J.C., Ferrando, P., Paiva, O., Lourenço, A., Cerezo, R. \& Valle, A. (2013). The relationship between approaches to teaching and approaches to studying: A two-level structural equation model for biology achievement in high school. Metacognition and learning, 8, 44-77. https://doi.org/10.1007/s11409013-9095-6

Ruiz, B., Trillos, J. y Morales, J. (2006). Estilos de aprendizaje y rendimiento académico en estudiantes universitarios. Revista Galego-portuguesa de Psicoloxía e Educación, 13, pp. 441-460. 
Torrano, M. y González Torres, A. (2004) http://www.webdocente.altascapacidades.es/Aprendizaje\%20Autorregulado/A rt 3 27.pdf

Zimmerman, B. (2002). Becoming a self regulated Learner: An Overview. Theory into practice, $41(2)$, https://doi.org/10.1207/s15430421tip4102 2

Zimmerman, B. J. (2000). Attainment of self-regulation: A social cognitive perspective. In M. Boekaerts, P. R. Pintrich \& M. Zeidner (Eds.), Handbook of selfregulation (pp. 13-39). San Diego, CA: Academic Press.

Zimmerman, B. y Schunk, D. (2001). Self-regulated learning and academic achievement: Theoretical perspectives (2aㅡ ed.). Nueva York: Springer-Verlag.

Zusho, A., Pintrich, P. R., y Coppola, B. (2003). Skill and will: The role of motivation and cognition in the learning of college chemistry. International Journal of Science

Education, 25 , 1081-1. https://doi.org/10.1080/0950069032000052207 